\title{
Turing Test as a Defining Feature of AI-Completeness
}

Roman V. Yampolskiy

\begin{abstract}
The paper contributes to the development of the theory of AICompleteness by formalizing the notion of AI-Complete and AI-Hard problems. The intended goal is to provide a classification of problems in the field of General Artificial Intelligence. We prove Turing Test to be an instance of an AI-Complete problem and further show certain AI problems to be AI-Complete or AI-Hard via polynomial time reductions. Finally, the paper suggests some directions for future work on the theory of AI-Completeness.
\end{abstract}

Keywords: AI-Complete, AI-Easy, AI-Hard, Human Oracle.

\section{Introduction}

Since its inception in the 1950s the field of Artificial Intelligence has produced some unparalleled accomplishments while at the same time failing to formalize the problem space it is concerned with. This paper proposes to address this shortcoming by extends on the work in [56] and contributing to the theory of AICompleteness, a formalism designed to do for the field of AI what notion of NP-Completeness did for computer science in general. It is our belief that such formalization will allow for even faster progress in solving remaining problems in humankind's conquest to build an intelligent machine.

According to the encyclopedia Wikipedia the term "AI-Complete" was proposed by Fanya Montalvo in the 1980s [54]. A somewhat general definition of the term included in the 1991 Jargon File [37] states:

“AI-Complete: [MIT, Stanford, by analogy with 'NP-complete'] adj. Used to describe problems or subproblems in AI, to indicate that the solution presupposes a solution to the 'strong AI problem' (that is, the synthesis of a human-level intelligence). A problem that is AI-complete is, in other words, just too hard. Examples

Roman V. Yampolskiy

Computer Engineering and Computer Science, DC 215,

University of Louisville, KY 40292

e-mail: roman.yampolskiy@louisville.edu

X.-S. Yang (Ed.): Artif. Intell., Evol. Comput. and Metaheuristics, SCI 427, pp. 3-17. springerlink.com

(C) Springer-Verlag Berlin Heidelberg 2012 
of AI-complete problems are 'The Vision Problem', building a system that can see as well as a human, and 'The Natural Language Problem', building a system that can understand and speak a natural language as well as a human. These may appear to be modular, but all attempts so far (1991) to solve them have foundered on the amount of context information and 'intelligence' they seem to require."

As such, the term "AI-Complete" (or sometimes AI-Hard) has been a part of the field for many years and has been frequently brought up to express difficulty of a specific problem investigated by researchers (see [31, 26, 15, 36, 6, 20, 32, $33,10,27,28,29,16,23,55])$. This informal use further encouraged similar concepts to be developed in other areas of science: Biometric-Completeness [36], ASR-Complete [30]. While recently numerous attempts to formalize what it means to say that a problem is "AI-Complete" have been published [2, 41, 11] even before such formalization attempts systems which relied on humans to solve problems which were perceived to be AI-Complete were utilized:

- AntiCaptcha systems use humans to break CAPTCHA security protocol [2, $58,59,63]$ either by directly hiring cheap workers in developing countries [5] or by rewarding correctly solved CAPTCHAs with presentation of pornographic images [52].

- Chinese Room philosophical argument by John Searle shows that including a human as a part of a computational system may actually reduce its perceived capabilities such as understanding and consciousness [40].

- Content Development online projects such as Encyclopedias (Wikipedia, Conservapedia), Libraries (Project Gutenberg, Video collections (YouTube) and Open Source Software (SourceForge) all rely on contributions from people for content production and quality assurance.

- Cyphermint a check cashing system relies on human workers to compare a snapshot of a person trying to perform a financial transaction to a picture of a person who initially enrolled with the system. Resulting accuracy outperforms any biometric system and is almost completely spoof proof (see cyphermint.com for more info).

- Data Tagging systems entice user into providing meta-data for images, sound or video files. A popular approach involves developing an online game which as a byproduct of participation produces a large amount of accurately labeled data [1].

- Distributed Proofreaders employs a number of human volunteers to eliminate errors in books created by relying on Optical Character Recognition process. (see pgdp.net for more info).

- Interactive Evolutionary Computation algorithms use humans in place of a fitness function to make judgments regarding difficult to formalize concept such as esthetic beauty or taste [47].

- Mechanical Turk is an Amazon.com's attempt at creating Artificial Intelligence. Humans are paid varying amounts for solving problems which are believed to be beyond current abilities of AI programs (see mturk.com for more 
info). The general idea behind the Turk has a broad appeal and the researchers are currently attempting to bring it to the masses via the Generalized Task Markets (GTM) [42, 19, 18, 21].

- Spam Prevention is easy to accomplish by having humans vote on emails they receive as spam or not. If a certain threshold is reached a particular piece of email could be said to be spam with a high degree of accuracy [13].

Recent work has attempted to formalize the intuitive notion of AI-Completeness. In particular three such endowers are worth reviewing [56]:

In 2003 Ahn et al. [2] attempted to formalize the notion of an AI-Problem and the concept of AI-Hardness in the context of computer security. An AI-Problem was defined as a triple: " $\mathcal{P}=(S, D, f)$, where $\mathrm{S}$ is a set of problem instances, $\mathrm{D}$ is a probability distribution over the problem set $\mathrm{S}$, and $\mathrm{f}: \mathrm{S} \rightarrow\{0 ; 1\}^{*}$ answers the instances. Let $\delta \in(0 ; 1]$. We require that for an $\alpha>0$ fraction of the humans $\mathrm{H}, \operatorname{Pr}_{\mathrm{x}_{\leftarrow} \mathrm{D}}[\mathrm{H}(\mathrm{x})=\mathrm{f}(\mathrm{x})]>\delta \ldots$ An AI problem $\mathcal{P}$ is said to be $(\delta, \tau)$-solved if there exists a program $\mathrm{A}$, running in time at most $\tau$ on any input from $\mathrm{S}$, such that $\operatorname{Pr}_{\mathrm{x} \in \mathrm{D}, \mathrm{r}}\left[\mathrm{A}_{\mathrm{r}}(\mathrm{x})=\mathrm{f}(\mathrm{x})\right] \geq \delta$. (A is said to be a $(\delta, \tau)$ solution to $\mathcal{P}$.) $\mathcal{P}$ is said to be a $(\delta, \tau)$-hard AI problem if no current program is a $(\delta, \tau)$ solution to $\mathcal{P}$, and the AI community agrees it is hard to find such a solution." It is interesting to observe that the proposed definition is in terms of democratic consensus by the AI community. If researchers say the problem is hard, it must be so. Also, time to solve the problem is not taken into account. The definition simply requires that some humans be able to solve the problem [2].

In 2007 Shahaf and Amir [41] have published their work on the Theory of AICompleteness. Their paper presents the concept of the Human-Assisted Turing Machine and formalizes the notion of different Human Oracles (see Section on Human Oracles for technical details). Main contribution of the paper comes in the form of a method for classifying problems in terms of human-versus-machine effort required to find a solution. For some common problems such as Natural Language Understanding (NLU) the paper proposes a method of reductions allowing conversion from NLU to the problem of Speech Understanding via Text-ToSpeech software.

In 2010 Demasi et al. [11] presented their work on problem classification for Artificial General Intelligence (AGI). The proposed framework groups the problem space into three sectors:

- Non AGI-Bound: Problems that are of no interest to AGI researchers.

- AGI-Bound: Problems that require human level intelligence to be solved.

- AGI-Hard: Problems that are at least as hard as any AGI Bound problem.

The paper also formalizes the notion of Human Oracles and provides a number of definitions regarding their properties and valid operations. 


\section{The Theory of AI-Completeness}

From people with mental disabilities to geniuses human minds are cognitively diverse and it is well known that different people exhibit different mental abilities. We define a notion of a Human Oracle (HO) function capable of computing any function computable by the union of all human minds. In other words any cognitive ability of any human being is repeatable by our HO. To make our Human Oracle easier to understand we provide the following illustration of the Human function:

String Human (String input) \{

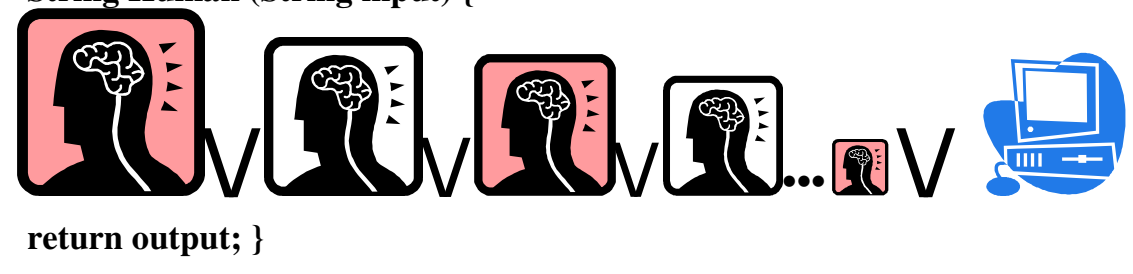

Fig. 1 Human oracle: Human ${ }_{\text {Best }}-$ a union of minds

Such a function would be easy to integrate with any modern programming language and would require that the input to the function be provided as a single string of length $N$ and the function would return a string of length $M$. No specific encoding is specified for the content of strings $N$ or $M$ and so they could be either binary representations of data or English language phrases both being computationally equivalent. As necessary the human function could call regular TM functions to help in processing of data. For example, a simple computer program which would display the input string as a picture to make human comprehension easier could be executed. Humans could be assumed to be cooperating perhaps because of a reward. Alternatively, one can construct a Human function which instead of the union of all minds computes the average decision of all human minds on a problem encoded by the input string as the number of such minds goes to infinity. To avoid any confusion we propose naming the first HO Human Best $_{\text {and }}$ and

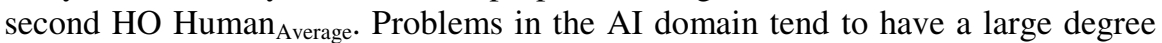
of ambiguity in terms of acceptable correct answers. Depending on the problem at hand the simplistic notion of an average answer could be replaced with an aggregate answer as defined in the Wisdom of Crowds approach [46]. Both functions could be formalized as Human-Assisted Turing Machines [41].

Human function is an easy to understand and use generalization of the Human Oracle. One can perceive it as a way to connect and exchange information with a real human sitting at a computer terminal. While easy to intuitively understand, such description is not sufficiently formal. Shahaf et al. have formalized the notion of Human Oracle as an HTM [41]. In their model a human is an oracle machine that can decide a set of languages $L_{i}$ in constant time: $H \subseteq\left\{L_{i} \mid L_{i} \subseteq \sum^{*}\right\}$. If time complexity is taken into account answering a question might take a non-constant time: $H \subseteq\left\{<L_{i}, f_{i}>\mid L_{i} \subseteq \sum^{*}, f_{i}: \mathbb{N} \rightarrow \mathbb{N}\right\}$ there $f_{i}$ is the time-complexity function 
for language $L_{i}$, meaning the human can decide if $\mathrm{x} \in L_{i}$ in $f_{i}(|\mathrm{x}|)$ time. In order to realistically address capabilities of individual humans a probabilistic oracle was also presented which provided correct answers with probability $p: H \subseteq\left\{<L_{i}, p_{i}>\mid\right.$ $\left.L_{i} \subseteq \sum^{*}, 0 \leq p_{i} \leq 1\right\}$. Finally the notion of reward is introduced into the model to capture humans improved performance on "paid" tasks: $H \subseteq\left\{<L_{i}, u_{i}\right\rangle \mid L_{i} \subseteq \sum^{*}, u_{i}$ $: \mathbb{N} \rightarrow \mathbb{N}\}$ where $u_{i}$ is the utility function [41].

\subsection{Definitions}

Definition 1: A problem $C$ is AI-Complete if it has two properties:

1. It is in the set of AI problems (Human Oracle solvable).

2. Any AI problem can be converted into $C$ by some polynomial time algorithm.

Definition 2: AI-Hard: A problem $H$ is AI-Hard if and only if there is an AIComplete problem $\mathrm{C}$ that is polynomial time Turing-reducible to $\mathrm{H}$.

Definition 3: AI-Easy: The complexity class AI-easy is the set of problems that are solvable in polynomial time by a deterministic Turing machine with an oracle for some AI problem. In other words, a problem X is AI-easy if and only if there exists some AI problem $\mathrm{Y}$ such that $\mathrm{X}$ is polynomial-time Turing reducible to $\mathrm{Y}$. This means that given an oracle for $\mathrm{Y}$, there exists an algorithm that solves $\mathrm{X}$ in polynomial time.

Figure 2 illustrates relationship between different AI complexity classes. Right side illustrates the situation if it is ever proven that AI-problems = AI-Complete problems. Left side shows the converse.

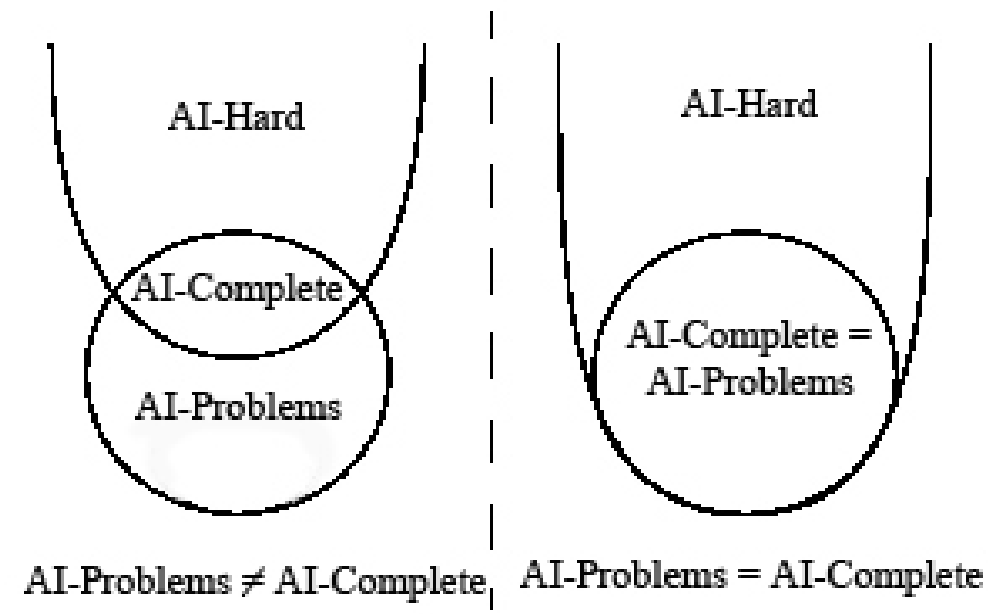

Fig. 2 Relationship between AI complexity classes 


\subsection{Turing Test as the First AI-Complete Problem}

In this section we will show that a Turing Test [50] problem is AI-Complete. First we need to establish that Turing Test is indeed an AI problem (HO solvable). This trivially follows from the definition of the test itself. The test measures if a human-like performance is demonstrated by the test taker and Human Oracles are defined to produce human level performance. While both "human" and "intelligence test" are intuitively understood terms we have already shown that Human Oracles could be expressed in strictly formal terms. The Turing Test itself also could be formalized as an interactive proof $[45,8,44]$.

Second requirement for a problem to be proven to be AI-Complete is that any other AI problem should be convertible into an instance of the problem under consideration in polynomial time via Turing reduction. Therefore we need to show how any problem solvable by the Human function could be encoded as an instance of a Turing Test. For any HO-solvable problem $h$ we have a String input which encodes the problem and a String output which encodes the solution. By taking the input as a question to be used in the TT and output as an answer to be expected while administering a TT we can see how any HO-solvable problem could be reduced in polynomial time to an instance of a Turing Test. Clearly the described process is in polynomial time and by similar algorithm any AI problem could be reduced to TT. It is even theoretically possible to construct a complete TT which utilizes all other problems solvable by HO by generating one question from each such problem.

\subsection{Reducing Other Problems to TT}

Having shown a first problem (Turing Test) to be AI-Complete the next step is to see if any other well-known AI-problems are also AI-complete. This is an effort similar to the work of Richard Carp who has shown some 21 problems to be NPComplete in his 1972 paper and by doing so started a new field of Computational Complexity [22]. According to the Encyclopedia of Artificial Intelligence [43] published in 1992 the following problems are all believed to be AI-Complete and so will constitute primary targets for our effort of proving formal AICompleteness on them [43]:

- Natural Language Understanding - "Encyclopedic knowledge is required to understand natural language. Therefore, a complete Natural Language system will also be a complete Intelligent system."

- Problem Solving - "Since any area investigated by AI researchers may be seen as consisting of problems to be solved, all of AI may be seen as involving Problem Solving and Search".

- Knowledge Representation and Reasoning - “...the intended use is to use explicitly stored knowledge to produce additional explicit knowledge. This is what reasoning is. Together Knowledge representation and Reasoning can be seen to be both necessary and sufficient for producing general intelligence - it is another AI-complete area." 
- Vision or Image Understanding - "If we take "interpreting" broadly enough, it is clear that general intelligence may be needed to do this interpretation, and that correct interpretation implies general intelligence, so this is another AI-complete area."

Now that Turing Test has been proven to be AI-Complete we have an additional way of showing other problems to be AI-Complete. We can either show that a problem is both in the set of AI problems and all other AI problem can be converted into it by some polynomial time algorithm or we can reduce any instance of Turing Test problem (or any other already proven to be AI-Complete problem) to an instance of a problem we are trying to show to be AI-Complete. This second approach seems to be particularly powerful. The general heuristic of our approach is to see if all information which encodes the question which could be asked during administering of a Turing Test could be encoded as an instance of a problem in question and likewise if any potential solution to that problem would constitute an answer to the relevant Turing Test question. Under this heuristic it is easy to see that for example Chess is not AI-Complete as only limited information can be encoded as a starting position on a standard size chess board. Not surprisingly Chess has been one of the greatest successes of AI and currently Chess playing programs dominate all human players including world champions.

Question Answering (QA) [17, 38] is a sub-problem in Natural Language Processing. Answering question at a level of a human is something HOs are particularly good at based on their definition. Consequently QA is an AI-Problem which is one of the two requirements for showing it to be AI-Complete. Having access to an Oracle capable of solving QA allows us to solve TT via a simple reduction. For any statement $S$ presented during administration of TT transform said statement into a question for the QA Oracle. The answers produced by the Oracle can be used as replies in the TT allowing the program to pass the Turing Test. It is important to note that access to the QA oracle is sufficient to pass the Turing Test only if questions are not restricted to stand alone queries, but could contain information from previous questions. Otherwise the problem is readily solvable even by today's machines such as IBM's Watson which showed a remarkable performance against human Jeopardy champions [35].

Speech Understanding (SU) [4] is another sub-problem in Natural Language Processing. Understanding Speech at a level of a human is something HOs are particularly good at based on their definition. Consequently SU is an AI-Problem which is one of the two requirements for showing it to be AI-Complete. Having access to an Oracle capable of solving SU allows us to solve QA via a simple reduction. We can reduce QA to SU by utilizing any Text-to-Speech software [49, 9] which is both fast and accurate. This reduction effectively transforms written questions into the spoken ones making it possible to solve every instance of QA by referring to the $\mathrm{SU}$ oracle.

\subsection{Other Probably AI-Complete Problems}

Figure 3 shows the relationship via reductions between problems shown to be AIComplete in this paper. We hope that our work will challenge the AI community to prove other important problems as either belonging or not belonging to that 
class. While the following problems have not been explicitly shown to be AIComplete, they are strong candidates for such classification and are also problems of great practical importance making their classification a worthy endower. If a problem has been explicitly conjectured to be AI-Complete in a published paper we include a source of such speculation: Dreaming [38], Commonsense Planning [41], Foreign Policy [26], Problem Solving [43], Judging a Turing Test [41], Common Sense Knowledge [3], Speech Understanding [41], Knowledge Representation and Reasoning [43], Word Sense Disambiguation [10, 32], Machine Translation [54], Ubiquitous Computing [23], Change Management for Biomedical Ontologies [33], Natural Language Understanding [43], Software Brittleness [54], Vision or Image Understanding [43].

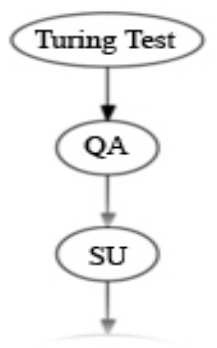

Fig. 3 Reductions from the first NP-Complete problem

\section{$2.51^{\text {st }}$ AI-Hard Problem: Programming}

We define the problem of Programming as taking a natural language description of a program and producing a source code which then compiled on some readily available hardware/software produces a computer program which satisfies all implicit and explicit requirements provided in the natural language description of the programming problem assignment. Simple examples of Programming are typical assignments given to students in computer science classes. Ex. "Write a program to play Tic-Tac-Toe." with successful students writing source code which if correctly compiled allows the grader to engage the computer in an instance of that game. Many requirements of such assignment remain implicit such as that response time of the computer should be less than a minute. Such implicit requirements are usually easily inferred by students who have access to culture instilled common sense. As of this writing no program is capable of solving Programming outside of strictly restricted domains.

Having access to an Oracle capable of solving Programming allows us to solve TT via a simple reduction. For any statement $S$ presented during TT transform said statement into a programming assignment of the form: "Write a program which would respond to $S$ with a statement indistinguishable from a statement provided by an average human" (A full transcript of the TT may also be provided for disambiguation purposes). Applied to the set of all possible TT statements this procedure clearly allows us to pass TT, however Programming itself is not in 
AI-Problems as there are many instances of Programming which are not solvable by Human Oracles. For example "Write a program to pass Turing Test" is not known to be an AI-Problem under the proposed definition. Consequently, Programming is an AI-Hard problem.

\section{Beyond AI-Completeness}

The human oracle function presented in this paper assumes that the human being behind it has some assistance from the computer in order to process certain human unfriendly data formats. For example a binary string representing a video is completely impossible for a human being to interpret but could easily be played by a computer program in the intended format making it possible for a human to solve a video understanding related AI-Complete problem. It is obvious that a human being provided with access to a computer (perhaps with Internet connection) is a more powerful intelligence compared to an unenhanced in such a way human. Consequently it is important to limit help from a computer to a human worker inside a human Oracle function to assistance in the domain of input/output conversion but not beyond as the resulting function would be both AI-Complete and "Computer Complete".

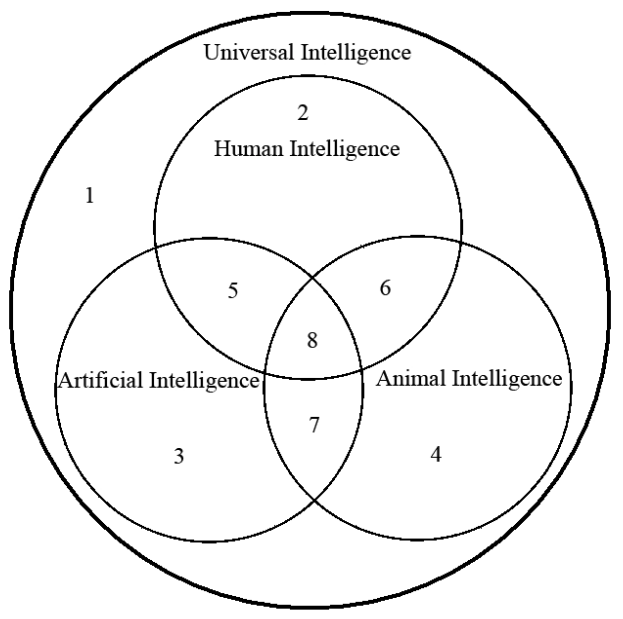

Fig. 4 Venn diagram for four different types of intelligence

Figure 4 utilizes a Venn diagram to illustrate subdivisions of problem space produced by different types of intelligent computational devices. Region 1 represents what is known as a Universal Intelligence [25] or a Super Intelligence [24, 61, 57, 60] a computational agent which outperforms all other intelligent agents over all possible environments. Region 2 is the standard unenhanced Human level intelligence of the type capable of passing a Turing Test, but at the same time incapable of computation 
involving large numbers or significant amount of memorization. Region 3 is what is currently possible to accomplish via the state-of-the-art AI programs. Finally Region 4 represents an abstract view of animal intelligence. AI intelligence researchers strive to produce Universal Intelligence and it is certainly likely to happen given recent trends in both hardware and software developments and theoretical underpinning of the Church/Turing Thesis [51]. It is also likely that if we are able to enhance human minds with additional memory and port those to a higher speed hardware we will essentially obtain a Universal Intelligence [39].

While the Universal Intelligence incorporates abilities of all the lower intelligences it is interesting to observe that Human, AI and Animal intelligences have many interesting regions of intersection [62]. For example animal minds are as good as human minds at visual understanding of natural scenes. Regions 5, 6, and 7 illustrate common problem spaces between two different types of intelligent agents. Region 8 represents common problem solving abilities of humans, computers and animals. Understanding such regions of commonality may help us to better separate involved computational classes which are represented by abilities of a specific computational agent minus the commonalities with a computational agent with which we are trying to draw a distinction. For example CAPTCHA [2] type tests rely on inability of computers to perform certain pattern recognition tasks with the same level of accuracy as humans to separate AI agents from Human agents. Alternatively a test could be devised to tell humans not armed with calculators from AIs by looking at the upper level of ability. Such a test should be easy to defeat once an effort is made to compile and formalize limitations and biases of the human mind.

It is also interesting to consider the problem solving abilities of hybrid agents. We have already noted that a human being equipped with a computer is a lot more capable compared to an unaided person. Some recent research in Brain Computer Interfaces [53] provides a potential path for future developments in the area. Just as interestingly combining pattern recognition abilities of animals with symbol processing abilities of AI could produce a computational agent with a large domain of human like abilities (see work on RoboRats [48] on monkey controlled robots [34]). It is very likely that in the near future the different types of intelligent agents will combine to even greater extent. While such work is under way we believe that it may be useful to introduce some additional terminology into the field of problem classification. For the complete space of problems we propose that the computational agents which are capable of solving a specific subset of such problems get to represent the set in question. Therefore we propose additional terms: "Computer-Complete" and "Animal-Complete" to represent computational classes solvable by such agents. It is understood that just like humans differ in their abilities so do animal and computers. Aggregation and averaging utilized in our human function could be similarly applied to definition of respective oracles. As research progre $\equiv$ ommon names may be needed for different combinations of regions from Figure 8 illustrating such concepts as Human-AI hybrid or Animal-Robot hybrid.

Certain aspects of human cognition do not map well onto the space of problems which have seen a lot of success in the AI research field. Internal states of human mind such as consciousness (stream of), self-awareness, understanding, emotions (love, hate), feelings (pain, pleasure), etc. are not currently addressable by our methods. Our current state-of-the-art technologies are not sufficient to unambiguously 
measure or detect such internal states and consequently even their existence is not universally accepted. Many scientists propose ignoring such internal states or claim they are nothing but a byproduct of flawed self-analysis. Such scientists want us to restrict science only to measurable behavioral actions, however since all persons have access to internal states of at least one thinking machine interest in trying to investigate internal states of human mind is unlikely to vanish.

While we were able to present a formal theory of AI-Completeness based on the concept of Human Oracles the theory is not strong enough to address problems involving internal states of mind. In fact one of the fundamental arguments against our ability to implement understanding in a system which is based on symbol manipulation, Searle's Chinese Room thought experiment, itself relies on a generalized concept of a human as a part of a computational cycle. It seems that the current Turing/Von Neumann architecture is incapable of dealing with the set of problems which are related to internal states of human mind. Perhaps a new type of computational architecture will be developed in the future capable of mimicking such internal states. It is likely that it will be inspired by a better understanding of human biology and cognitive science. Research on creating Artificial Consciousness (AC) is attracting a lot of attention at least in terms of number of $\mathrm{AC}$ papers published.

As a part of our ongoing effort to classify AI related problems we propose a new category specifically devoted to problems of reproducing internal states of a human mind in artificial ways. We call this group of problems ConsciousnessComplete or C-Complete for short. An oracle capable of solving C-Complete problems would be fundamentally different from the Oracle Machines proposed by Turing. C-Oracles would take input in the same way as their standard counterparts but would not produce any symbolic output. The result of their work would be a novel internal state of the oracle, which may become accessible to us if the new type of hardware discussed above is developed.

Just like SAT was shown to be the first NP-Complete problem and Turing Test to be the first AI-Complete problem we suspect that Consciousness will be shown to be the first $\mathrm{C}$-Complete problem with all other internal-state related problems being reducible to it. Which of the other internal state problems are also C-Complete is beyond the scope of this preliminary work. Even with no consciousness-capable hardware available at the moment of this writing the theory of C-Completeness is still a useful tool as it allows for formal classification of classical problems in the field of Artificial Intelligence into two very important categories: potentially solvable (with current technology) and unsolvable (with current technology). Since the only information available about Human Oracles is their output and not internal states they are fundamentally different from C-Oracles creating two disjoint sets of problems.

History of AI research is full of unwarranted claims of anticipated breakthroughs and conversely overestimations regarding difficulty of some problems. Viewed through the prism of our AI-Complete/C-Complete theories history of AI starts to make sense. Solutions for problems which we classify as AI-Complete have been subject to continues steady improvement while those falling in the realm of C-Completeness have effectively seen zero progress (Computer Pain [7, 12], Artificial Consciousness [40, 14], etc.). To proceed science needs to better 
understand what is the difference between a feeling and a though is. Feeling pain and knowing about pain are certainly not the same internal states. We are hopefully that the future research in this area will bring some long awaited answers.

\section{Conclusions}

Progress in the field of artificial intelligence requires access to well defined problems of measurable complexity. The theory of AI-Completeness aims to provide a base for such formalization. Showing certain problems to be AI-Complete/-Hard is useful for developing novel ways of telling computers from humans. Also, any problem shown to be AI-Complete would be a great alternative way of testing an artificial intelligent agent to see if it attained human level intelligence [41].

\section{References}

[1] Ahn, L.V.: Games With A Purpose. IEEE Computer Magazine, 96-98 (June 2006)

[2] Ahn, L.V., Blum, M., Hopper, N., Langford, J.: CAPTCHA: Using Hard AI Problems for Security. In: Biham, E. (ed.) EUROCRYPT 2003. LNCS, vol. 2656, pp. 294-311. Springer, Heidelberg (2003)

[3] Andrich, C., Novosel, L., Hrnkas, B.: Common Sense Knowledge, Exercise Paper Information Search and Retrieval (2009),

http://www.iicm.tu-graz.ac.at/cguetl/courses/isr/ uearchive/uews2009/Ue06-CommonSenseKnowledge.pdf

[4] Anusuya, M.A., Katti, S.K.: Speech Recognition by Machine: A Review. International Journal of Computer Science and Information Security (IJCSIS) 6(3), 181-205 (2009)

[5] Bajaj, V.: Spammers Pay Others to Answer Security Tests. The New York Times (April 25, 2010)

[6] Bergmair, R.: Natural Language Steganography and an "AI-complete" Security Primitive. In: 21st Chaos Communication Congress, Berlin (December 2004)

[7] Bishop, M.: Why Computers Can't Feel Pain. Minds and Machines 19(4), 507-516 (2009)

[8] Bradford, P.G., Wollowski, M.: A formalization of the Turing Test. SIGART Bulletin 6(4), 3-10 (1995)

[9] Chan, T.-Y.: Using a Text-to-Speech Synthesizer to Generate a Reverse Turing Test. In: 15th IEEE International Conference on Tools with Artificial Intelligence (ICTAI 2003), p. 226 (2003)

[10] Chen, J., Liu, J., Yu, W., Wu, P.: Combining Lexical Stability and Improved Lexical Chain for Unsupervised Word Sense Disambiguation. In: Second International Symposium on Knowledge Acquisition and Modeling (KAM 2009), Wuhan, November 30, pp. 430-432 (2009)

[11] Demasi, P., Szwarcfiter, J.L., Cruz, A.J.O.: A Theoretical Framework to Formalize AGI-Hard Problems. In: The Third Conference on Artificial General Intelligence, Lugano, Switzerland, March 5-8 (2010)

[12] Dennett, D.C.: Why You Can't Make a Computer That Feels Pain. Synthese 38(3), 415-456 (1978) 
[13] Dimmock, N., Maddison, I.: Peer-to-peer collaborative spam detection. Crossroads 11(2) (December 2004)

[14] Dreyfus, H.L.: What computers can't do; A critique of artificial reason. Harper \& Row (1972)

[15] Gentry, C., Ramzan, Z., Stubblebine, S.: Secure distributed human computation. In: 6th ACM Conference on Electronic Commerce, pp. 155-164 (2005)

[16] Hendler, J.: We've Come a Long Way, Maybe .... IEEE Intelligent Systems 23(5), 23 (2008)

[17] Hirschman, L., Gaizauskas, R.: Natural Language Question Answering. The View from Here. Natural Language Engineering 7(4), 275-300 (2001)

[18] Horvitz, E.: Reflections on Challenges and Promises of Mixed-Initiative Interaction. AI Magazine-Special Issue on Mixed-Initiative Assistants 28(2) (2007)

[19] Horvitz, E., Paek, T.: Complementary Computing: Policies for Transferring Callers from Dialog Systems to Human Receptionists. User Modeling and User Adapted Interaction 17(1), 159-182 (2007)

[20] Ide, N., Véronis, J.: Introduction to the special issue on word sense disambiguation: the state of the art. Computational Linguistics 24(1), 1-40 (1998)

[21] Kapoor, A., Tan, D., Shenoy, P., Horvitz, E.: Complementary Computing for Visual Tasks: Meshing Computer Vision with Human Visual Processing. In: IEEE International Conference on Automatic Face and Gesture Recognition (2008)

[22] Karp, R.M.: Reducibility Among Combinatorial Problems. In: Miller, R.E., Thatcher, J.W. (eds.) Complexity of Computer Computations, pp. 85-103. Plenum, NY (1972)

[23] Leahu, L., Sengers, P., Mateas, M.: Interactionist AI and the promise of ubicomp, or, how to put your box in the world without putting the world in your box. In: Tenth International Conference on Ubiquitous Computing, Seoul, South Korea, September 2124, pp. 1-10 (2008)

[24] Legg, S.: Machine Super Intelligence. PhD Thesis, University of Lugano (June 2008), http: / /www. vetta.org/documents / Machine_Super_Intelligence.pdf

[25] Legg, S., Hutter, M.: Universal Intelligence: A Definition of Machine Intelligence. Minds and Machines 17(4), 391-444 (2007)

[26] Mallery, J.C.: Thinking About Foreign Policy: Finding an Appropriate Role for Artificially Intelligent Computers. Annual Meeting of the International Studies Association. St. Louis, MO (1988)

[27] Mcintire, J.P., Havig, P.R., Mcintire, L.K.: Ideas on authenticating humanness in collaborative systems using AI-hard problems in perception and cognition. In: IEEE National Aerospace \& Electronics Conference, Dayton, OH, July 21-23, pp. 50-55 (2009)

[28] Mcintire, J.P., Mcintire, L.K., Havig, P.R.: A variety of automated turing tests for network security: Using AI-hard problems in perception and cognition to ensure secure collaborations. In: International Symposium on Collaborative Technologies and Systems (CTS 2009), Baltimore, MD, May 18-22, pp. 155-162 (2009)

[29] Mert, E., Dalkilic, C.: Word sense disambiguation for Turkish. In: 24th International Symposium on Computer and Information Sciences (ISCIS 2009), Guzelyurt, September 14-16, pp. 205-210 (2009) 
[30] Morgan, N., Baron, D., Bhagat, S., Carvey, H., Dhillon, R., Edwards, J., Gelbart, D., Janin, A., Krupski, A., Peskin, B., Pfau, T., Shriberg, E., Stolcke, A., Wooters, C.: Meetings about meetings: research at ICSI on speech in multiparty conversations. In: IEEE International Conference on Acoustics, Speech, and Signal Processing (ICASSP 2003), April 6-10 (2003)

[31] Mueller, E.T.: Daydreaming and Computation. Ph.D. Dissertation, University of California, Los Angeles, p. 302 (March 1987)

[32] Navigli, R., Velardi, P.: Structural Semantic Interconnections: A Knowledge-Based Approach to Word Sense Disambiguation. IEEE Transactions on Pattern Analysis and Machine Intelligence 27(7), 1075-1086 (2005)

[33] Nejad, A.S.: A Framework for Analyzing Changes in Health Care Lexicons and Nomenclatures. PhD dissertation. Concordia University, Quebec, Canada (April 2010)

[34] Nicolelis, M.A.L., Wessberg, J., Stambaugh, C.R., Kralik, J.D., Beck, P.D., Laubach, M., Chapin, J.K., Kim, J.: Real-time prediction of hand trajectory by ensembles of cortical neurons in primates. Nature 408(6810), 361 (2000)

[35] Pepitone, J.: IBM's Jeopardy supercomputer beats humans in practice bout, CNNMoney, http: //money.cnn.com/2011/01/13/ technology/ibm_jeopardy_watson (retrieved on January 13, 2011)

[36] Phillips, P.J., Beveridge, J.R.: An introduction to biometric-completeness: The equivalence of matching and quality. In: IEEE 3rd International Conference on Biometrics: Theory, Applications, and Systems (BTAS 2009), Washington, DC, September 28-30, pp. 1-5 (2009)

[37] Raymond, E.S.: Jargon File Version 2.8.1 (March 22, 1991), http: / / catb.org/esr/jargon/oldversions/jarg282.txt

[38] Salloum, W.: A Question Answering System based on Conceptual Graph Formalism. In: The 2nd International Symposium on Knowledge Acquisition and Modeling (KAM 2009), China, November 30 (2009)

[39] Sandberg, A., Boström, N.: Whole Brain Emulation: A Roadmap, Future of Humanity Institute, Oxford University. Technical Report \#2008-3 (2008), http: / /www. fhi.ox.ac.uk/Reports/2008-3.pdf

[40] Searle, J.: Minds, Brains and Programs. Behavioral and Brain Sciences 3(3), 417-457 (1980)

[41] Shahaf, D., Amir, E.: Towards a theory of AI completeness. In: 8th International Symposium on Logical Formalizations of Commonsense Reasoning (Commonsense 2007), California, March 26-28 (2007)

[42] Shahaf, D., Horvitz, E.: Generalized Task Markets for Human and Machine Computation. In: Twenty-Fourth AAAI Conference on Artificial Intelligence, Atlanta, GA (July 2010)

[43] Shapiro, S.C.: Artificial Intelligence. In: Shapiro, S.C. (ed.) Encyclopedia of Artificial Intelligence, pp. 54-57. John Wiley, New York (1992)

[44] Shieber, S.M.: Does the Turing Test demonstrate intelligence or not. In: Twenty-First National Conference on Artificial Intelligence (AAAI 2006), Boston, MA, July 16-20 (2006)

[45] Shieber, S.M.: The Turing Test as Interactive Proof. Nous 41(4), 686-713 (2007)

[46] Surowiecki, J.: The Wisdom of Crowds: Why the Many Are Smarter Than the Few and How Collective Wisdom Shapes Business, Economies, Societies and Nations, Little, Brown (2004)

[47] Takagi, H.: Interactive Evolutionary Computation: Fusion of the Capacities of EC Optimization and Human Evaluation. Proc. of the IEEE 89(9), 1275-1296 (2001) 
[48] Talwar, S.K., Xu, S., Hawley, E.S., Weiss, S.A., Moxon, K.A., Chapin, J.K.: Behavioural neuroscience: Rat navigation guided by remote control. Nature 417, 37-38 (2002)

[49] Taylor, P., Black, A.: Speech synthesis by phonological structure matching. In: Eurospeech 1999, Budapest, Hungary, pp. 1531-1534 (1999)

[50] Turing, A.: Computing Machinery and Intelligence. Mind 59(236), 433-460 (1950)

[51] Turing, A.M.: On Computable Numbers, with an Application to the Entscheidungsproblem. Proceedings of the London Mathematical Society 42, 230-265 (1936)

[52] Vaas, L.: Striptease Used to Recruit Help in Cracking Sites. PC Magazine (December 1, 2007)

[53] Vidal, J.: Toward direct brain-computer communication. Annual Review of Biophysics and Bioengineering 2, 157-180 (1973)

[54] Wikipedia, AI-Complete, http: //en.wikipedia.org/wiki/AI-complete (retrieved January 7, 2011)

[55] Yampolskiy, R.V.: AI-Complete CAPTCHAs as Zero Knowledge Proofs of Access to an Artificially Intelligent System. ISRN Artificial Intelligence, 271878 (2011)

[56] Yampolskiy, R.V.: AI-Complete, AI-Hard, or AI-Easy - Classification of Problems in AI. In: The 23rd Midwest Artificial Intelligence and Cognitive Science Conference, Cincinnati, OH, USA, April 21-22 (2012) (under Review)

[57] Yampolskiy, R.V.: Artificial Intelligence Safety Engineering: Why Machine Ethics is a Wrong Approach. In: Philosophy and Theory of Artificial Intelligence (PT-AI 2011), Thessaloniki, Greece, October 3-4 (2011)

[58] Yampolskiy, R.V.: Embedded CAPTCHA for Online Poker. In: 20th Annual CSE Graduate Conference (Grad.-Conf. 2007), Buffalo, NY (2007)

[59] Yampolskiy, R.V.: Graphical CAPTCHA embedded in cards. In: Western New York Image Processing Workshop (WNYIPW), IEEE Signal Processing Society, Rochester (2007)

[60] Yampolskiy, R.V.: Leakproofing Singularity - Artificial Intelligence Confinement Problem. Journal of Consciousness Studies (JCS) 19(1-2) (2012)

[61] Yampolskiy, R.V.: What to Do with the Singularity Paradox? In: Philosophy and Theory of Artificial Intelligence (PT-AI 2011), Thessaloniki, Greece, October 3-4 (2011)

[62] Yampolskiy, R.V., Fox, J.: Artificial Intelligence and the Human Mental Model. In: Eden, A., Moor, J., Soraker, J., Steinhart, E. (eds.) In the Singularity Hypothesis: a Scientific and Philosophical Assessment. Springer (2012)

[63] Yampolskiy, R.V., Govindaraju, V.: Embedded Non-Interactive Continuous Bot Detection. ACM Computers in Entertainment 5(4), 1-11 (2007) 